\title{
ASSOCIATION OF DEPRESSION WITH SEXUAL AND DAILY ACTIVITIES: A COMMUNITY STUDY OF OCOTOGENARIAN MEN
}

Osvaldo P. Almeida ${ }^{1,2,3}$

Bu B. Yeap ${ }^{4,5}$

Graeme J. Hankey ${ }^{4,6}$

Jonathan Golledge ${ }^{7}$

Leon Flicker ${ }^{2,4,8}$

${ }^{1}$ School of Psychiatry \& Clinical Neurosciences, University of Western Australia, Perth, Western Australia, Australia

${ }^{2}$ Western Australian Centre for Health \& Ageing, Centre for Medical Research, University of Western Australia, Perth, Western Australia, Australia

${ }^{3}$ Department of Psychiatry, Royal Perth Hospital, Perth, Western Australia, Australia

${ }^{4}$ School of Medicine and Pharmacology, University of Western Australia, Perth, Western Australia, Australia

${ }^{5}$ Department of Endocrinology and Diabetes, Fremantle Hospital, Fremantle, Western Australia, Australia

${ }^{6}$ Department of Neurology, Royal Perth Hospital, Perth, Western Australia, Australia

${ }^{7}$ Queensland Research Centre for Peripheral Vascular Disease, School of Medicine and Dentistry, James Cook University, Townsville, Australia

${ }^{8}$ Department of Geriatric Medicine, Royal Perth Hospital, Perth, Western Australia, Australia

Word-count: 3489 (excluding references and tables)

Author Contributions

Conceived and designed the experiments: Almeida.

Performed the experiments: Almeida, Norman, Flicker and Hankey.

Analyzed the data: Almeida.

Wrote the manuscript: Almeida.

Other: All authors reviewed the manuscript for important intellectual content and approved its submission for publication.

Declaration of interest: No disclosures to report.

Funding/Support: National Health and Medical Research Council of Australia (NHMRC).

Role of the Sponsors: The sponsors had no role in the design and conduct of the study; collection, management, analysis, and interpretation of the data; or preparation, review, or approval of the manuscript.

\section{Correspondence}

Prof. Osvaldo P. Almeida, WA Centre for Health \& Ageing (M573), University of Western Australia, 35 Stirling Highway, Crawley, Perth, WA 6009, Australia. Email: osvaldo.almeida@uwa.edu.au. 


\section{ABSTRACT}

Objective: To determine the association between clinically significant depressive symptoms, routine function and sexual interest and practice in a community-derived sample of octogenarian men.

Design and methods: Cross-sectional study of 1649 community dwelling men aged 80 years or over with no history of terminal illnesses or neurodegenerative diseases. Men with Patient Health Questionnaire (PHQ-9) scores $\geq 10$ were deemed to be clinically depressed. Scores between 5 and 9 were considered indicative of subthreshold depression. We used standard procedures to collect self-reported sociodemographic, lifestyle and clinical data, as well as basic and instrumental activities of daily living, and a structured questionnaire to ask men about their 12-month interest in sex, frequency, past experiences, and current sexual problems.

Results: 121 men (7.3\%) had clinically significant depression and 239 (14.5\%) subthreshold depression. Depressive symptoms were associated with difficulties in basic and instrumental activities of daily living, but not with sexual practice. Decreased interest in sex and anxiety before sex were associated with subthreshold depression. Clinically significant depressive symptoms were independently and positively associated with past history of diabetes (odds ratio, OR=2.1, $95 \% \mathrm{Cl}=1.1,4.0)$, depression $(\mathrm{OR}=9.0 ; 95 \% \mathrm{Cl}=4.6,17.3)$, impaired ability to groom (OR=3.7, $95 \% \mathrm{Cl}=1.2,11.0)$, carry out heavy housework duties $(\mathrm{OR}=2.4,95 \% \mathrm{Cl}=1.1-5.1)$, manage finances $(\mathrm{OR}=2.5,95 \% \mathrm{Cl}=1.1-5.7)$ or engage in leisure activities $(\mathrm{OR}=4.1,95 \% \mathrm{Cl}=2.0-8.2)$. Conclusions: Ability to function effectively at home, financial autonomy and leisure are associated with clinically significant depression in octogenarian men. Maintaining daily function and autonomy may be suitable a target for interventions that aim to reduce the prevalence and incidence of depression in older age. 


\section{INTRODUCTION}

Symptoms of depression are common across the lifespan, although some epidemiological evidence suggests that their prevalence declines in later life. ${ }^{1} \mathrm{O}^{\prime}$ Connor has argued that the proportion of people with depression increases with age and that findings in the opposite direction are a product of biased sampling strategies and case ascertainment, as older disabled people are often excluded from health surveys and structured interviews that rely on the use of skip questioning fail to ascertain the presence of all relevant symptoms of depression. ${ }^{2}$

Findings from the Precipitating Events Project (PEP), which investigated the health outcomes of 754 people older than 70 years of age over a period of 108 months, showed that severe or chronic disability doubled the odds of depression during follow up. ${ }^{3}$ Data from a sample of Taiwanese people aged 65 years or older suggested that severe or chronic disability may be a direct cause of depression. ${ }^{4}$ Disability is more frequent in late than early life, and is particularly common among those aged 80 years or over. ${ }^{55}$ This is the segment of the population that has been growing the fastest worldwide, ${ }^{6}$ which suggests that the overall prevalence of depression may also increase as the population ages. Whether specific types of functional impairment, such as the ability to groom without assistance or to manage one's own financial affairs, are more closely related to depression in later life remains to be determined.

Concerns about ascertainment bias in previous studies of depression arise from the common use of two simple screening questions (about low or depressed mood and decreased interest or enjoyment in doing things) to determine whether to pursue a more detailed assessment of depressive symptoms. ${ }^{7}$ An affirmative answer to either of these questions triggers further questioning about other depressive symptoms, such as changes in sleep or appetite, decreased 
energy, psychomotor slowing or agitation, impaired concentration, ideas of guilt or failure, and suicidal thoughts. Older adults who are depressed often deny dysphoric mood, ${ }^{8}$ and this may lead to a substantial decrease in the sensitivity of screening procedures in this age group. Moreover, questions designed to ascertain the ability to experience enjoyment tend to avoid direct enquiry about certain pleasurable activities in late life, such as sex. We have previously reported that sexual activity occurs less frequently among older men with a past history of clinically significant depressive symptoms or use of antidepressants, ${ }^{9}$ which suggests that a systematic sexual history may add valuable information to the routine assessment of depression, particularly in older men.

We designed the present study to determine the independent risk factors for clinically significant depressive symptoms in a community-derived sample of men aged 80 years or over, with a particular focus on daily function and sexual interest and practice. The underlying rationale behind our approach is that sexual activity represents yet another aspect of the routine function of older people. Establishing such associations is important because many functional and sexual problems are amenable to intervention, and their management could potentially contribute to enhance quality of life and decrease the prevalence of depression among octogenarians.

\section{METHODS}

\section{Ethics statement}

The study was conducted according to the principles expressed in the Declaration of Helsinki. The Human Research Ethics Committee of the University of Western Australia approved the study protocol and all men provided written informed consent to participate.

\section{Study design and participants}


This study used a population-based sample of older men living in the Perth metropolitan area, the Health In Men Study (HIMS). Details regarding the recruitment of participants have been described elsewhere. ${ }^{10}$ Briefly, we recruited a community-representative sample of 19352 Australian men aged 65 to 85 years living in the Perth metropolitan area between 1996 and 1998, of whom 12203 completed the first assessment of HIMS, which was originally conceived to investigate the effect of screening for abdominal aortic aneurysm on mortality. ${ }^{10}$ In 20087319 men were still alive and were invited to complete a new self-rating postal questionnaire, of whom 173 could not be contacted, 230 were too unwell to take part, 361 withdrew consent, and 3281 did not respond, leaving a total sample of 3274 participants. Of these, 388 failed to complete the Patient Health Questionnaire, 38 reported a diagnosis of dementia and a further 32 of Parkinson's disease. Finally, for the purposes of this study, another 1167 men were excluded because they were younger than 80 years, leaving a total study sample of 1649 octogenarian men.

\section{Outcome of interest: clinically significant depressive symptoms}

We used the Patient Health Questionnaire (PHQ-9) to assess depressive symptoms. ${ }^{11}$ The PHQ-9 consists of nine questions about how often the respondent has been bothered by depressive symptoms during the past two weeks, and each item can be scored 0 ('not at all'), 1 ('several days'), 2 ('a week or more') or 3 ('nearly every day'). A total score of 10 or more indicates the presence of clinically significant depression. ${ }^{12}$ Men with PHQ-9 total score between 5 and 9 (inclusive) were deemed to display subthreshold symptoms of depression. Individual PHQ-9 items were considered present when participants reported that the relevant symptom had been present for at least 'a week or more' during the preceding two weeks.

\section{Explanatory variables}


We calculated the age of participants as the difference in days between the date of the assessment and their date of birth divided by 365.25. In addition, men provided information about their country of birth (Australian vs overseas) as well as the highest level of education achieved (completed or not high school). Marital status was recorded as 'married' if men reported being married or that they were living in a de facto relationship. Participants also recorded whether they were living alone or with others, and provided information about their lifestyle. They were considered physically inactive if they reported less than 150 minutes of total activity during the past week: brisk walking, moderate to vigorous leisure activities (tennis, swimming or other activities associated with 'puff and pant'), or vigorous household chores. We also collected information about smoking (never, past and current), and the consumption of alcohol, fruits and vegetables. Men who reported consuming 15 or more standard drinks per week on a regular basis were considered to be using alcohol at a harmful level. ${ }^{131313}$

Participants were asked (yes/no) whether they had ever been told by a doctor that they had hypertension, diabetes, dyslipidemia (high levels cholesterol, lipids, triglycerides or fats in the blood), coronary heart disease (angina or previous heart attack), stroke, prostate cancer, bowel cancer, skin cancer or depression (including age when first told). They also listed all medications they were consuming at the time of assessment, and we used the World Health Organisation Anatomical Therapeutic Chemical (ATC) classification system to determine if participants were consuming antidepressants (codes N06A), anxiolitics (codes N05B) and hypnotics (codes N05C) (World Health Organisation Collaborating Centre for Drug Statistics Methodology, Oslo, Norway, 2005). 
Men rated their functional limitations in grooming, eating normal food, bathing or taking a shower, dressing upper body, dressing lower body, getting up from a chair, walking inside the house, using the toilet, shopping for personal items or groceries, doing light housework (e.g., washing dishes, dusting), doing heavy housework, laundry, managing money, preparing main meals, taking medications, using the telephone, and doing leisure activities or hobbies. ${ }^{14,15}$ Possible answers for each question were: no difficulty, some difficulty, major difficulty, unable to do without help. For the purposes of this study, we considered that men who indicate having 'major difficulty' or of being 'unable to do without help' showed evidence of impaired function in the relevant area. Men also indicated whether they had been receiving formal assistance from a health or social agency during the 6 months before assessment (yes/no) in the areas of food services or preparation, domestic services, home maintenance, personal care, and respite services.

Finally, we asked participants to indicate whether during the preceding 12 months they had 'lacked interest in having sex' (yes/no), 'how often' they had had sex (did not have sex / at least once per week / two to three times per week / once a month or less), and about their lifetime sexual experiences (women only, women and men, men only, celibate). For those who reported being sexually active (i.e., had sex at least once during the past year), we asked them to indicate (yes/no) any problems they might have experienced, including inability to reach climax (orgasm), premature ejaculation, pain during the intercourse, anxiety before having sex, and trouble maintaining an erection. Possible answers to these questions were 'not at all' versus 'at least some of the time'.

\section{$\underline{\text { Statistical analyses }}$}


Data were managed and analyzed with the statistical package Stata release 12.1 (StataCorp, College Station, TX). We used descriptive statistics (mean, standard deviation of the mean [SD], proportions) to summarize our data, and compared the distribution of sociodemographic, lifestyle, clinical, functional and sexual behavior information of men with (PHQ-9 $\geq 10$ ) and without depression using the Wilcoxon rank test ( $\chi^{2}$ statistic for ordinal numerical variables) and Pearson's chi-square statistic ( $\chi^{2}$ for categorical variables). We used Bonferroni correction following multiple comparisons between subgroups (i.e., not depressed vs subthreshold depression, not depressed vs depression, subthreshold depression vs depression). Variables associated with depression in univariate analyses $(p<0.1)$ were forced into a multivariate logistic regression explanatory model that had depression status as its dependent variable. Finally, we determined whether access to formal social support services influenced the association between explanatory variables and depression. Odds ratios (OR) were reported with their associated $95 \%$ confidence interval $(95 \% \mathrm{Cl})$. We used Wald $\chi^{2}$ statistic following logistic regression to report the effect of certain exposures (e.g., inability to perform heavy housework duties: yes/no) on the presence of depression. Alpha was set at $5 \%$ and all tests reported are two-tailed and included information about the number of degrees of freedom (df).

\section{RESULTS}

The age of participants ranged from 80 to 93.7 years (mean=83.9, SD=3.0). One hundred and twenty-one men (7.3\%) showed evidence of clinically significant depressive symptoms, and 239 (14.5\%) met the study criteria for subthreshold depression. Their sociodemographic, lifestyle and clinical characteristics are summarized in table 1 . Men with depression were older than those without, and were also more likely to be living alone. Physical inactivity was more prevalent among men with than without depression, as was the frequency of diabetes, coronary heart 
disease, heart failure and stroke. The proportion of men using antidepressants and hypnotics was higher among men with than without depression, and so was the prevalence of a past diagnosis of depression. The distribution of these exposures among men with subthreshold depression fell between that of men without depression and participants with clinically significant depressive symptoms.

TABLE 1

Men with depression reported a much higher prevalence of restricted function than those without across all routine functions (Table 2). Again, the proportion of men with subthreshold depression experiencing functional limitations fell between that of men without depression and with clinically significant depressive symptoms. Lack of interest in sex was common in both men with and without depression (51.5\% of the entire sample), and was more frequent among men with subthreshold depressive symptoms than men without depression. The difference between men with clinically significant depressive symptoms and men without depression was not statistically significant (after Bonferroni correction). Sexual problems were common among older men who reported being sexually active, regardless of whether they were depressed or not. The most frequent complaints were difficulty maintaining an erection (69.9\%) and reaching an orgasm (48.4\%). The online supplementary table 1 describes the sociodemographic, lifestyle and clinical characteristics of participants who did and did not provide an answer to the questions assessing sexual function.

TABLE 2

TABLE 3 
Table 3 shows the odds ratio of subthreshold and clinically significant depressive symptoms compared with no depression according to exposures associated with the presence of depressive symptoms in univariate analyses $(p<0.1)$. Subthreshold depressive symptoms were associated with diabetes, skin cancer, past diagnosis of depression, and restricted ability to get up, complete heavy household chores, manage medications and use the telephone, as well as lack of interest in sex. Clinically significant depressive symptoms were associated with diabetes, past diagnosis of depression, and restricted ability to groom, carry out heavy household chores, manage finances and take part in leisure activities. Requiring assistance to eat was associated with decreased odds of clinically significant depression. The use of home assistance was reported by 632 men (38.3\%), 125 (52.3\%) of whom were experiencing subthreshold depression and 79 (65.3\%) clinically significant depression. Addition of this variable to the multivariate logistic regression model had little effect except that inability to perform heavy housework was no longer associated with clinically significant depression (Wald $\left.\chi^{2}(\mathrm{df}=1)=0.23, p=0.824\right)$.

As engagement in leisure activities and hobbies might be compromised by the presence of clinically significant depressive symptoms, particularly decreased interest or ability to experience pleasure, we re-ran our logistic regression model and over-adjusted it for the presence of anhedonia (i.e., we forced this item into the model even though it contributes to the PHQ-9 total score). Impaired ability to engage in leisure activities remained associated with increased odds of clinically significant depression despite this over-adjustment (Wald $\left.\chi^{2}(d f=1)=5.67, p=0.017\right)$.

The use of antidepressants was not independently associated with decreased sexual interest in this sample once other relevant exposures were included in the model $\left(\right.$ Wald $\chi^{2}(d f=1)=0.09$, 
$p=0.765$ ). Similarly, being married was not independently associated with sexual interest (Wald $\left.\chi^{2}(d f=1)=0.43, p=0.510\right)$ or activity $\left(\right.$ Wald $\left.\chi^{2}(d f=1)=0.24, p=0.623\right)$ in this sample.

\section{DISCUSSION}

This study reports novel data on a very large sample of community dwelling octogenarian men ( $n=1649)$, of whom 121 (7.3\%) showed evidence of clinically significant symptoms of depression and 239 of subthreshold depressive symptoms. We found that impairment in any activity of daily living (either basic or instrumental) was associated with increased odds of subtreshold and clinically significant depressive symptoms in univariate analyses, but lack of sexual practice was not. Multivariate analysis showed that only past history of diabetes, depression, and difficulties with grooming, heavy household chores, finances and engaging in leisure activities were associated with clinically significant depressive symptoms. An overlapping, but not entirely similar, risk profile was identified for subthreshold depressive symptoms: history of diabetes, skin cancer or past depression, getting up unassisted, heavy household chores, managing medications, using the phone and lack of interest in sex. Before discussing the relevance and implication of these findings, we will consider the characteristics of the study design and how these might affect the interpretation of the results and their generalizability.

\section{$\underline{\text { Limitations }}$}

Establishing causal relationships is always difficult in observational studies, particularly when findings are based on cross-sectional data. We found that clinically significant depressive symptoms were independently associated with self-reported history of past depressive episodes, diabetes, and impairment in leisure activities and management of finances. One could argue that 
loss of independence in these instrumental activities of daily living contributes to the causal pathway that leads to depression, but the reverse seems equally feasible: depression could lead to impaired routine function. Currently available evidence indicates that disability is a stronger predictor of depression than depressive symptoms are of disability, ${ }^{4}$ and our adjusted analyses are consistent with such an interpretation (e.g., the association between impaired ability to engage in leisure activities could not be explained by lack of interest or reduce ability to experience pleasure). It is also possible that the relationship between functional impairment and depression is bi-directional, with functional impairment increasing the risk of depressive symptoms, which in turn might increase functional impairment and lead to further worsening or perpetuation of depressive symptoms.

This study was limited to men and we cannot be certain that our findings would apply equally to women, as expectations regarding ageing and routine function may vary according to gender. ${ }^{16}$ Another limitation to consider is that $70 \%$ of our octogenarians were aged 80 to 84 , which limited our ability to adjust the analyses for the effect of increasing age. Consequently, our findings apply to a predominantly "young" group of very older men. Healthy participant bias is also likely to have occurred, as significantly disabled persons would be less able or willing to complete the assessment than study subjects. Indeed, the results of past analyses confirm that this has occurred in our sample. ${ }^{10,17}$ The potential consequence of such a bias would be loss of power and inability to declare as significant meaningful associations between certain functional impairments and depression. In fact, our finding that men who required assistance to eat had lower adjusted odds of depression was most probably due to the small number of men with depression who reported this type of impairment $(n=11)$, so that statistical adjustments might have distorted the results. Moreover, we acknowledge that the study measures were based on self-reported information 
rather than a structured clinical assessment, although the scales used to diagnose clinically significant depressive symptoms and functional impairment have well-established facevalidity. ${ }^{12,15}$ Finally, about $14 \%$ of participants did not answer the questions about sexual activity this could potentially have affected the results of our analyses if missing data were not distributed at random in people with and without depression. We would suggest that such interpretation seems improbable for two reasons: (i) we modeled missing data in our analyses, (ii) the distribution of missing data among men with and without depression seems to have occurred at random.

\section{$\underline{\text { Interpretation of the results }}$}

The results of this study are based on a large community-derived sample of men aged 80 years or over for whom detailed information on sociodemographic, lifestyle, clinical and functional variables were available. This, together with our unique data on sexual behavior, has enabled us to explore for the first time the association between depression, routine function and sexual activity among very old men free of neurodegenerative conditions such as Alzheimer's and Parkinson's diseases (these conditions are known to affect routine function).

We found a point prevalence of clinically significant depressive symptoms of $7.3 \%$ (14.5\% for subthreshold depression), which suggests that depression may not be more frequent in community-dwelling men aged 80 years or over than in younger age groups. ${ }^{1,18}$ We also found that most men in this age group are no longer interested in sex and that the majority had not been sexually active during the preceding 12 months. There was an excess of loss of interest in sex among men with subthreshold depression, although there was no evidence that sexual inactivity was associated with greater odds of subthreshold or clinically significant depressive symptoms in 
the multivariate analyses. In addition, the proportion of men reporting sexual problems among those who had been sexually active during the past year was high, but had no obvious association with the presence of clinically significant depressive symptoms. However, more men with subthreshold depressive symptoms reported pre-sex anxiety than men without depression. Taken together, these results indicate that, in later life, sex may play a less prominent role in mental well being than in younger age groups. ${ }^{19}$

In contrast, measures of daily function across various domains were associated with prevalent depressive symptoms, the most critical areas being engagement in leisure activities, ability to carry out heavy household chores (e.g., manual jobs around the house) and manage one's own finances. A possible interpretation of these findings is that the perceived loss of financial autonomy and ability to engage in activities that are pleasing and meaningful to old men has a detrimental effect on mood. In other words, clinically significant depression in octogenarian men may be associated with decreased self-efficacy (self-efficacy represents one's perceived ability to complete the tasks required to achieve one's goals). ${ }^{20}$ Should such a relationship be causal, interventions that succeed in enhancing perceived self-efficacy might also contribute to decrease the prevalence of depression in men aged 80 years or older. Provision of services, such as home assistance, is important to maintain some level of independence, but might fail to address the perceived loss of autonomy that extend beyond basic daily requirements, as suggested by our data. There may be scope for provision of higher-level assistance to address issues of financial autonomy and opportunities for meaningful personal activities. Existing psychological interventions with established efficacy for the management of depression might prove useful in the management of mildly disabled old men, such as problem solving and behavioral activation. ${ }^{21}$ Societal factors, such 
respect and purposeful roles for older people, may also be important. Trial data are now required to confirm or dismiss such predictions.

Interestingly, the risk profile of men with subthreshold depressive symptoms overlapped but was not the same as that of men with clinically significant depression, which suggests they might represent a distinct group of older men. One possibility is that symptoms of anxiety might be more prominent in this group, as suggested by the presence of sex-related anxiety. Indeed, previous studies have shown that older people who display subsyndromal depressive symptoms are at greater risk of developing not only depression but also anxiety disorders. ${ }^{22}$

We found that past history of depression was associated with a higher risk of prevalent depression in our sample, a finding that is consistent with those of other studies. ${ }^{23}$ This may simply highlight the fact that depression is a recurrent disorder - as a consequence, past history of depression should be particularly common in older people with depression. Another possibility is that the observed association between current and past diagnosis of depression is due to greater persistence of depression in older age. ${ }^{24}$ These results highlight the need for vigorous management and ongoing monitoring of this population.

\section{Conclusion}

The results of this survey indicate that clinically significant depressive symptoms in men older than 80 years are associated with past history of depression and impaired ability to engage in leisure activities and manage one's own financial affairs. Sexual activity and interest were not associated with depression, but lack of interest in sex was associated with subthreshold depression. As the world's population ages, an increasingly larger proportion of people will live well into their 80 s and 
will require interventions that can adequately meet their needs and expectations. The design of future randomized trials aiming to reduce the prevalence and incidence of depression among octogenarians might beneficially consider the potential for behavioral interventions based on these findings. 


\section{REFERENCES}

1. Henderson AS, Jorm AF, Korten AE, et al: Symptoms of depression and anxiety during adult life: evidence for a decline in prevalence with age. Psychol Med 1998; 28:1321-1328.

2. O'Connor DW: Do older Australians truly have low rates of anxiety and depression? A critique of the 1997 National Survey of Mental Health and Wellbeing. Aust N Z J Psychiatry 2006; 40:623-631.

3. Barry LC, Soulos PR, Murphy TE, et al: Association between indicators of disability burden and subsequent depression among older persons. J Gerontol A Biol Sci Med Sci 2012;

4. Chen CM, Mullan J, Su YY, et al: The longitudinal relationship between depressive symptoms and disability for older adults: a population-based study. J Gerontol A Biol Sci Med Sci 2012; 67:1059-1067.

5. Australian Institute of Health and Welfare. Australia's health 2010. Australia's health series no. 12. Cat. no. AUS 122. Canberra: AlHW.,

6. Cohen JE: Human population: the next half century. Science 2003; 302:1172-1175.

7. Arroll $\mathrm{B}$, Khin N,Kerse $\mathrm{N}$ : Screening for depression in primary care with two verbally asked questions: cross sectional study. BMJ 2003; 327:1144-1146.

8. Gallo JJ, Rabins PV, Lyketsos CG, et al: Depression without sadness: functional outcomes of nondysphoric depression in later life. J Am Geriatr Soc 1997; 45:570-578.

9. Hyde Z, Flicker L, Hankey GJ, et al: Prevalence of sexual activity and associated factors in men aged 75 to 95 years: a cohort study. Ann Intern Med 2010; 153:693-702.

10. Norman PE, Flicker L, Almeida OP, et al: Cohort Profile: The Health In Men Study (HIMS). Int J Epidemiol 2009; 38:48-52.

11. Kroenke K, Spitzer RL,Williams JB: The PHQ-9: validity of a brief depression severity measure. J Gen Intern Med 2001; 16:606-613.

12. Arroll B, Goodyear-Smith F, Crengle S, et al: Validation of PHQ-2 and PHQ-9 to screen for major depression in the primary care population. Ann Fam Med 2010; 8:348-353.

13. National Health and Medical Research Council. Australian Guidelines to Reduce Health Risks from Drinking Alcohol. Commonwealth of Australia, Canberra, 2009.,

14. Katz $S$, Ford AB, Moskowitz RW, et al: Studies of illness in the aged. The index of ADL: a standardized measure of biological and psychosocial function. JAMA 1963; 185:914-919.

15. Lawton MP,Brody EM: Assessment of older people: self-maintaining and instrumental activities of daily living. Gerontologist 1969; 9:179-186. 
16. Melzer D, McWilliams B, Brayne C, et al: Socioeconomic status and the expectation of disability in old age: estimates for England. J Epidemiol Community Health 2000; 54:286-292.

17. Almeida OP, Yeap BB, Hankey GJ, et al: Low free testosterone concentration as a potentially treatable cause of depressive symptoms in older men. Arch Gen Psychiatry 2008; 65:283-289.

18. O'Connor DW,Parslow RA: Mental health scales and psychiatric diagnoses: responses to GHQ12, K-10 and CIDI across the lifespan. J Affect Disord 2010; 121:263-267.

19. Courtenay WH: Constructions of masculinity and their influence on men's well-being: a theory of gender and health. Soc Sci Med 2000; 50:1385-1401.

20. Strecher VJ, DeVellis BM, Becker $\mathrm{MH}$, et al: The role of self-efficacy in achieving health behavior change. Health Educ Q 1986; 13:73-92.

21. Cuijpers $P$, Geraedts AS, van Oppen $P$, et al: Interpersonal psychotherapy for depression: a meta-analysis. Am J Psychiatry 2011; 168:581-592.

22. van't Veer-Tazelaar PJ, van Marwijk HW, van Oppen P, et al: Prevention of late-life anxiety and depression has sustained effects over 24 months: a pragmatic randomized trial. Am J Geriatr Psychiatry 2011; 19:230-239.

23. Kendler KS, Gardner CO,Prescott CA: Toward a comprehensive developmental model for major depression in men. Am J Psychiatry 2006; 163:115-124.

24. Denihan A, Kirby $M$, Bruce I, et al: Three-year prognosis of depression in the communitydwelling elderly. Br J Psychiatry 2000; 176:453-457. 
Table 1. Distribution of sociodemographic, lifestyle and clinical variables among octogenarian men according to depression scores: no depression for $\mathrm{PHQ}-9<5$, subthreshold depression for $5 \leq \mathrm{PHQ}-$ $9<10$, and clinically significant depression for PHQ $-9 \geq 10$.

\begin{tabular}{|c|c|c|c|c|c|}
\hline & $\begin{array}{c}\text { No depression } \\
\mathrm{N}=1289 \\
\mathrm{n}(\%)\end{array}$ & $\begin{array}{l}\text { Subthreshold } \\
\qquad \begin{array}{c}\mathrm{N}=239 \\
\mathrm{n}(\%)\end{array} \\
\end{array}$ & $\begin{array}{c}\text { Depression } \\
\mathrm{N}=121 \\
\mathrm{n}(\%)\end{array}$ & $\begin{array}{l}\text { Statistic } \\
(\mathrm{df})^{*}\end{array}$ & $p$ \\
\hline \multicolumn{6}{|l|}{ Socio-demographic: } \\
\hline Age, mean (SD) years & $83.8(3.0)$ & $84.1(3.1)$ & $84.7(3.4)$ & $\chi^{2}(2)=10.31$ & 0.006 \\
\hline Overseas born & $654(50.7)$ & $124(51.9)$ & $59(48.8)$ & $\chi^{2}(2)=0.31$ & 0.855 \\
\hline Completed high school & 649 (50.3) & $125(52.3)$ & $56(46.3)$ & $\chi^{2}(2)=1.17$ & 0.558 \\
\hline Married & $919(71.3)$ & $170(71.1)$ & $73(60.3)$ & $\chi^{2}(4)=7.56$ & 0.109 \\
\hline Living alone & $305(23.7)$ & $58(24.3)$ & $40(33.1)$ & $\chi^{2}(4)=17.87$ & 0.001 \\
\hline \multicolumn{6}{|l|}{ Lifestyle: } \\
\hline Physically inactive & $479(37.2)$ & $129(54.0)$ & $83(68.6)$ & $\chi^{2}(2)=61.63$ & $<0.001$ \\
\hline Never smoked & $490(38.0)$ & $73(30.5)$ & $36(29.7)$ & $\chi^{2}(6)=12.14$ & 0.059 \\
\hline Past smoker & 699 (54.2) & $140(58.6)$ & $68(56.2)$ & & \\
\hline Current smoker & $33(2.6)$ & $8(3.3)$ & $6(5.0)$ & & \\
\hline Harmful alcohol use & 589 (45.7) & $127(53.1)$ & $60(49.6)$ & $\chi^{2}(4)=10.96$ & 0.027 \\
\hline Two or more serves of fruit per day & $860(66.7)$ & $155(64.8)$ & $70(57.8)$ & $\chi^{2}(4)=16.21$ & 0.003 \\
\hline Five or more serves of vegetables per day & $221(17.1)$ & $35(14.6)$ & $12(9.9)$ & $\chi^{2}(4)=21.80$ & $<0.001$ \\
\hline \multicolumn{6}{|l|}{ Clinical: } \\
\hline Hypertension & $684(53.1)$ & $132(55.2)$ & $68(56.2)$ & $\chi^{2}(4)=2.22$ & 0.696 \\
\hline Diabetes & $200(15.5)$ & $59(24.7)$ & $32(26.4)$ & $\chi^{2}(4)=30.21$ & $<0.001$ \\
\hline Dyslipidaemia & $468(36.3)$ & $96(40.2)$ & $52(43.0)$ & $\chi^{2}(4)=5.50$ & 0.240 \\
\hline Coronary heart disease & $260(20.2)$ & $64(26.8)$ & $50(41.3)$ & $\chi^{2}(4)=36.93$ & $<0.001$ \\
\hline Heart failure & $68(5.3)$ & $27(11.3)$ & $15(12.4)$ & $\chi^{2}(4)=23.67$ & $<0.001$ \\
\hline Stroke & $146(11.3)$ & $41(17.1)$ & $25(20.7)$ & $\chi^{2}(4)=13.45$ & 0.009 \\
\hline Prostate cancer & $184(14.3)$ & $39(16.3)$ & $26(21.5)$ & $\chi^{2}(4)=8.48$ & 0.075 \\
\hline Bowel cancer & $71(5.5)$ & $13(5.4)$ & $6(5.0)$ & $\chi^{2}(4)=2.54$ & 0.638 \\
\hline Skin cancer (including melanoma) & $584(45.3)$ & $121(50.6)$ & $51(42.1)$ & $\chi^{2}(4)=8.52$ & 0.074 \\
\hline Past diagnosis of depression & $62(4.8)$ & $43(18.0)$ & $40(33.1)$ & $\chi^{2}(4)=162.52$ & $<0.001$ \\
\hline Age first depressed $(n=142)^{\#}$, mean (SD) & $66.5(16.4)$ & $69.2(16.9)$ & $66.4(17.6)$ & $\chi^{2}(2)=1.24$ & 0.538 \\
\hline \multicolumn{6}{|l|}{ Psychotropic medications: } \\
\hline Antidepressant & $72(5.6)$ & $39(16.3)$ & $33(27.3)$ & $\chi^{2}(2)=85.46$ & $<0.001$ \\
\hline Anxiolytic & $19(1.5)$ & $10(4.2)$ & $4(3.3)$ & $\chi^{2}(2)=8.68$ & 0.013 \\
\hline Hypnotic & $41(3.2)$ & $25(10.5)$ & $17(14.0)$ & $\chi^{2}(2)=44.56$ & $<0.001$ \\
\hline
\end{tabular}

PHQ-9: Patient Health Questionnaire

df: number of degrees of freedom.

*Statistical tests included cells with missing values, hence $\mathrm{df} \geq 4$ for analyses when missing values were present.

${ }^{\#} 3$ men with history of depression did not report age of onset of symptoms. 
Table 2. Distribution of functional measures and sexual behavior among octogenarian men according to depression scores: no depression for $\mathrm{PHQ}-9<5$, questionable depression for $5 \leq \mathrm{PHQ}-$ $9<10$, and clinically significant depression for PHQ $-9 \geq 10$.

\begin{tabular}{|c|c|c|c|c|c|}
\hline & $\begin{array}{c}\text { No depression } \\
\mathrm{N}=1289 \\
\mathrm{n}(\%)\end{array}$ & $\begin{array}{c}\text { Subthreshold } \\
\mathrm{N}=239 \\
\mathrm{n}(\%)\end{array}$ & $\begin{array}{c}\text { Depression } \\
\mathrm{N}=121 \\
\mathrm{n}(\%)\end{array}$ & $\begin{array}{l}\text { Statistic } \\
(\mathrm{df})^{*}\end{array}$ & $\mathrm{p}$ \\
\hline \multicolumn{6}{|l|}{ Restricted routine function: } \\
\hline Grooming & 24 (1.9) & $26(10.9)$ & $25(20.7)$ & $\chi^{2}(4)=116.56$ & $<0.001$ \\
\hline Eating & $27(2.1)$ & $14(5.9)$ & $11(9.1)$ & $\chi^{2}(4)=26.37$ & $<0.001$ \\
\hline Bathing & $49(3.8)$ & $40(16.7)$ & $34(28.1)$ & $\chi^{2}(4)=131.39$ & $<0.001$ \\
\hline Dressing upper body & $88(6.8)$ & $42(17.6)$ & $37(30.6)$ & $\chi^{2}(4)=85.73$ & $<0.001$ \\
\hline Dressing lower body & $159(12.3)$ & $68(28.4)$ & $52(43.0)$ & $\chi^{2}(4)=101.45$ & $<0.001$ \\
\hline Getting up & $260(20.2)$ & $109(45.6)$ & $67(55.4)$ & $\chi^{2}(4)=125.01$ & $<0.001$ \\
\hline Walking around house & $76(5.9)$ & $51(21.3)$ & $47(38.8)$ & $\chi^{2}(4)=162.76$ & $<0.001$ \\
\hline Using toilet & $54(4.2)$ & $33(13.8)$ & $35(28.9)$ & $\chi^{2}(4)=115.61$ & $<0.001$ \\
\hline Purchasing groceries & $88(6.8)$ & $68(28.4)$ & $61(50.4)$ & $\chi^{2}(4)=241.75$ & $<0.001$ \\
\hline Light housework & $76(5.9)$ & $62(25.9)$ & $51(42.1)$ & $\chi^{2}(4)=205.44$ & $<0.001$ \\
\hline Heavy housework & $362(28.1)$ & $141(59.0)$ & $89(73.5)$ & $\chi^{2}(4)=199.54$ & $<0.001$ \\
\hline Managing finances & $81(6.3)$ & $40(16.7)$ & $39(32.2)$ & $\chi^{2}(4)=119.52$ & $<0.001$ \\
\hline Preparing meals & $225(17.5)$ & $91(38.1)$ & $59(48.8)$ & $\chi^{2}(4)=103.06$ & $<0.001$ \\
\hline Managing medications & $50(3.9)$ & $40(16.7)$ & $22(18.2)$ & $\chi^{2}(4)=95.14$ & $<0.001$ \\
\hline Using the telephone & $109(8.5)$ & $55(23.0)$ & $41(33.9)$ & $\chi^{2}(4)=120.45$ & $<0.001$ \\
\hline Leisure activities & $207(16.1)$ & $101(42.3)$ & $82(67.8)$ & $\chi^{2}(4)=238.97$ & $<0.001$ \\
\hline \multicolumn{6}{|l|}{ Sexual behavior: } \\
\hline Lack of interest in sex & $616(47.8)$ & $161(67.4)$ & $73(60.3)$ & $\chi^{2}(4)=36.78$ & $<0.001$ \\
\hline No sex during past year & 824 (63.9) & $159(66.5)$ & $84(69.4)$ & $\chi^{2}(4)=8.46$ & 0.076 \\
\hline $\begin{array}{r}\text { Past sexual experiences: } \begin{array}{r}\text { Celibate } \\
\text { Women only } \\
\text { Women and men } \\
\text { Men only } \\
\text { No response }\end{array}\end{array}$ & $\begin{array}{c}18(1.4) \\
1091(84.6) \\
6(0.5) \\
4(0.3) \\
170(13.2)\end{array}$ & $\begin{array}{c}9(3.8) \\
204(85.4) \\
3(1.3) \\
0 \\
23(9.6)\end{array}$ & $\begin{array}{c}6(5.0) \\
100(82.6) \\
0 \\
0 \\
115(12.4)\end{array}$ & $\chi^{2}(8)=17.60$ & 0.024 \\
\hline No response & \multicolumn{5}{|c|}{ For men sexually active during past year } \\
\hline Sexual problems: & $\mathrm{N}=295$ & $\mathrm{~N}=44$ & $\mathrm{~N}=16$ & & \\
\hline Unable to reach climax & $133(45.1)^{12}$ & $29(65.9)^{1}$ & $10(62.5)$ & $\chi^{2}(4)=8.33$ & 0.080 \\
\hline Premature ejaculation & $82(27.8)^{15}$ & $14(31.8)^{4}$ & $5(31.2)$ & $\chi^{2}(4)=2.70$ & 0.610 \\
\hline Pain during intercourse & $5(1.7)^{12}$ & $3(6.8)^{3}$ & $1(6.2)$ & $\chi^{2}(4)=6.52$ & 0.163 \\
\hline Pre-sex anxiety & $99(33.6)^{15}$ & $29(65.9)^{2}$ & $7(43.7)$ & $\chi^{2}(4)=18.28$ & 0.001 \\
\hline Trouble maintaining erection & $202(68.5)^{9}$ & $35(79.5)^{2}$ & $11(68.7)$ & $\chi^{2}(4)=3.82$ & 0.432 \\
\hline
\end{tabular}

df: number of degrees of freedom.

*Statistical tests included cells with missing values, hence $d f \geq 4$ for analyses when missing values were present.

${ }^{\mathrm{n}}=$ Number of men for whom information was missing. 
Table 3. Odds ratio of subthreshold depression and of depression derived from a multinomial logistic regression analysis that included demographic, lifestyle, and clinical and functional factors. All variables associated with either subthreshold depression or with depression $(p<0.1)$ were forced into the model.

\begin{tabular}{|c|c|c|}
\hline & $\begin{array}{c}\text { Subthreshold depression } \\
\text { Odds Ratio }(95 \% \mathrm{Cl})\end{array}$ & $\begin{array}{c}\text { Depression } \\
\text { Odds Ratio }(95 \% \mathrm{Cl}) \\
\end{array}$ \\
\hline Age, in years & $0.95(0.89,1.01)$ & $1.00(0.92,1.10)$ \\
\hline Living alone & $1.08(0.72,1.63)$ & $1.69(0.92,3.09)$ \\
\hline Physically inactive & $1.03(0.70,1.50)$ & $1.68(0.92,3.08)$ \\
\hline \multirow[t]{3}{*}{ Smoking history } & $1.16(0.79,1.71)$ & $0.90(0.49,1.67)$ \\
\hline & $1.14(0.37,3.53)$ & $0.54(0.10,2.95)$ \\
\hline & $1.25(0.88,1.77)$ & $1.05(0.60,1.82)$ \\
\hline Two or more server of fruit per day & $1.16(0.79,1.70)$ & $0.86(0.48,1.55)$ \\
\hline Five or more vegetables per day & $0.99(0.61,1.60)$ & $0.60(0.23(1.55)$ \\
\hline Diabetes & $1.98(1.31,2.99)$ & $2.12(1.13,3.98)$ \\
\hline Coronary heart disease & $1.19(0.78,1.82)$ & $1.75(0.92,3.32)$ \\
\hline Heart failure & $1.34(0.71,2.53)$ & $0.94(0.33,2.66)$ \\
\hline Stroke & $0.99(0.56,1.54)$ & $1.04(0.49,2.21)$ \\
\hline Prostate cancer & $0.93(0.56,1.54)$ & $1.39(0.68,2.84)$ \\
\hline Skin cancer & $1.53(1.06,2.20)$ & $0.78(0.43,1.39)$ \\
\hline Past diagnosis of depression & $3.47(2.06,5.87)$ & $8.97(4.64,17.33)$ \\
\hline Restricted function: & $2.22(0.88,5.59)$ & $3.68(1.23,10.98)$ \\
\hline Eating & $0.42(0.13,1.31)$ & $0.16(0.04,0.65)$ \\
\hline Bathing & $1.36(0.61,3.02)$ & $2.05(0.77,5.51)$ \\
\hline Dressing upper body & $0.52(0.25,1.10)$ & $0.48(0.19,1.23)$ \\
\hline Dressing lower body & $1.06(0.60,1.88)$ & $0.95(0.43,2.12)$ \\
\hline Getting up & $1.96(1.29,2.98)$ & $0.98(0.48,2.02)$ \\
\hline Walking around house & $1.12(0.58,2.14)$ & $1.69(0.71,4.02)$ \\
\hline Using toilet & $0.64(0.29,1.45)$ & $1.57(0.60,4.12)$ \\
\hline Purchasing groceries & $1.09(0.56,2.12)$ & $2.11(0.89,4.96)$ \\
\hline Light housework & $1.81(0.92,3.57)$ & $0.68(0.27,1.72)$ \\
\hline Heavy housework & $1.88(1.22,2.90)$ & $2.36(1.10,5.07)$ \\
\hline Managing finances & $1.06(0.55,2.04)$ & $2.49(1.08,5.72)$ \\
\hline Preparing meals & $1.45(0.92,2.27)$ & $1.01(0.50,2.05)$ \\
\hline Managing medications & $2.11(1.08,4.13)$ & $0.89(0.32,2.49)$ \\
\hline Using the telephone & $1.82(1.09,3.03)$ & $1.63(0.78,3.38)$ \\
\hline Leisure activities & $0.97(0.60,1.55)$ & $4.06(2.01,8.17)$ \\
\hline Lack of interest in sex & $2.26(1.43,3.59)$ & $1.26(0.64,2.50)$ \\
\hline No sex during past year & $0.70(0.43,1.15)$ & $0.56(0.25,1.28)$ \\
\hline
\end{tabular}

$95 \% \mathrm{Cl}$ : $95 \%$ confidence interval of the odds ratio.

Note: Inability to reach sexual climax and pre-sex anxiety were not included in the model because of the large number of missing points caused by the low number of older men who had engaged in sexual activities during the preceding year. 